\title{
Chronic Subdural Hematoma, a Cause of Persistent Post-Dural Puncture Headache in the Postpartum Period
}

\author{
Daniel Kanyata1, Omar Ahmed Nassir², Christopher K. Musau², Victoria Adhiambo Gamba ${ }^{3}$, \\ Mariatu Tamimu3 ${ }^{3}$ Michelle Gathecha1, Lily Nyamai4 \\ ${ }^{1}$ Directorate of Clinical Services, Kenyatta University Hospital, Nairobi, Kenya \\ ${ }^{2}$ Department of Surgery, School of Medicine, University of Nairobi, Nairobi, Kenya \\ ${ }^{3}$ Department of Obstetrics and Gynaecology, School of Medicine, University of Nairobi, Nairobi, Kenya \\ ${ }^{4}$ Department of Special Surgery, Kenyatta University, Kahawa, Kenya \\ Email: danielkanyata@gmail.com, ahmednasir99@yahoo.com,drckmusau@gmail.com,gambavick@gmail.com, \\ tamimu985@gmail.com
}

How to cite this paper: Kanyata, D., Nassir, O.A., Musau, C.K., Gamba, V.A., Tamimu, M., Gathecha, M. and Nyamai, L. (2021) Chronic Subdural Hematoma, a Cause of Persistent Post-Dural Puncture Headache in the Postpartum Period. Open Journal of Obstetrics and Gynecology, 11, 272-278. https://doi.org/10.4236/ojog.2021.113026

Received: September 21, 2020

Accepted: March 15, 2021

Published: March 18, 2021

Copyright $\odot 2021$ by author(s) and Scientific Research Publishing Inc. This work is licensed under the Creative Commons Attribution International License (CC BY 4.0).

http://creativecommons.org/licenses/by/4.0/ (c) (i) Open Access

\begin{abstract}
Background: Chronic subdural heamatoma (CSDH) is a disease that predominantly occurs in the elderly population. This is because of physiological atrophy of the brain parenchyma and frailty which leads to higher risk of falls. $\mathrm{CSDH}$ is unusual in the younger population but can be seen in the context of impact injuries in the younger population and mostly in males. Case Reports: We describe CSDH in 4 young postpartum mothers with no history of trauma. All had cesarean section births under spinal anaesthesia. The 4 mothers' ages range from 24 years to 32 years. They presented with persistent post-dural puncture headaches with 2 of the mothers having focal neurological deficits. They presented between 4 weeks and 6 weeks after cesarean section. Three mothers underwent burrhole evacuation with one mother having a craniotomy. All recovered after surgery. Conclusion: Spinal anaesthesia and disruption of the CSF dynamics is the only identifiable risk factors in these young mothers. A longitudinal follow-up of mothers undergoing spinal anaesthesia should be done to document the incidence of $\mathrm{CSDH}$ in postpartum mothers.
\end{abstract}

\section{Keywords}

Case Report, Post Dural Puncture Headache

\section{Introduction}

Chronic subdural heamatoma (CSDH) is a disease that predominantly occurs in 
the elderly population. This is because of physiological atrophy of the brain parenchyma and frailty which leads to higher risk of falls.

$\mathrm{CSDH}$ is unusual in the younger population but can be seen in the context of impact injuries in the younger population and mostly in males.

The internal pressure dynamics of the skull contents are determined by a constant balance of all its elements. Changes in the CSF pressure dynamics have been shown to predispose the individual to the development of CSDH. Iatrogenic CSF diversion in coexisting hydrocephalus or lumbar drainage can cause $\mathrm{CSDH}$.

CSDH after cesarean section under spinal anesthesia has been described in a few case studies and is considered rare.

We describe 4 postpartum women with $\mathrm{CSDH}$ who we have managed within 1 year and describe their characteristics.

\section{Cases}

\subsection{Case Report 1}

A 24-year-old primiparous woman at 4 weeks post-partum was imaged at a peripheral hospital for persistent headaches then sent to our facility for neurosurgical consult. Her primary complains were headache, that first started $48 \mathrm{hrs}$ after delivery by caesarean section with use of spinal anaesthesia. There was no mention of the gauge of spinal needle used at time of delivery. The patient had an uneventful pregnancy up-to delivery when she was operated on for poor progress of labour as reported by the mother. She had no history of previous pregnancies or abortions or hypertension during her pregnancy.

She describes her headaches as global and worse on any sudden movements. Exacerbated by coughing and later in the disease would wake her up in the middle of the night. She had intermittent visual blurring and some nausea. She used various pain medications including the opioid tramadol but with no good relief necessitating a visit at the peripheral hospital where the clinician ordered a CT scan.

Her examination was unremarkable with no focal deficits detected on examination. Her routine blood works showed normal platelet count and INR of 1.29.

The CT-Scan showed bilateral Chronic subdural hematoma (Figure 1).

The hematomas were evacuated through a left sided craniotomy and two right sided burr holes with rinsing of the hematoma cavity with normal saline until the effluent was clear. Postoperative course was uneventful with repeat CT Scan 5 months later showing resolution of hematoma (Figure 2).

\subsection{Case Report 2}

A 29-year old woman presented with constant headaches $48 \mathrm{hrs}$ after caesarean section under spinal anaesthesia for her third pregnancy. The indications for surgery were antepartum haemorrhage at 34 weeks gestation in a two previous scar patient. 


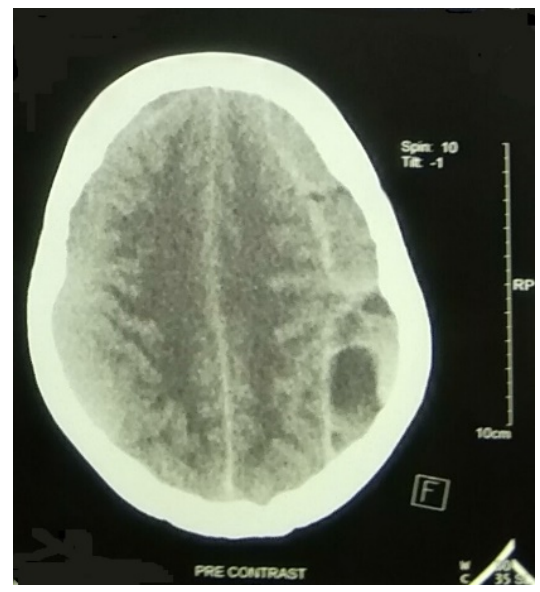

Figure 1. Bilateral chronic subdural hematomas with the left sided hematomas showing membranes.

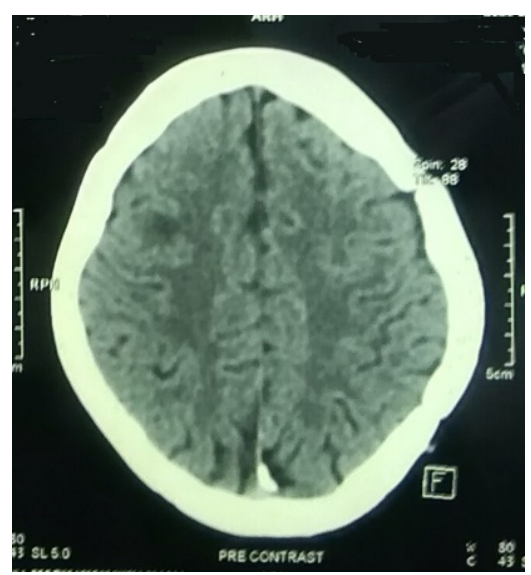

Figure 2. Post-operative images 5 months later showing resolution of hematoma after surgery.

The headaches which were global, were of sudden onset and constant with no diurnal variation. They were exacerbated in the standing and bending position with radiation to the side of the neck. She reported relief in the recumbent position and no response strong analgesia. She had associated right upper limb weakness, nausea and vomiting. She also developed double vision two weeks prior to admission (Figure 3 ).

She had no loss of consciousness, convulsions or hypertension during her last pregnancy. Her antenatal profile was negative for HIV and syphilis.

Her $1^{\text {st }}$ pregnancy in 2011 was complicated by severe hypertension in pregnancy at 26 weeks but no medication was given, Emergency Caesarean Section under General Anaesthesia was performed due to Antepartum haemorrhage. The hypertension resolved 1-month postpartum. In her $2^{\text {nd }}$ pregnancy emergency caesarean section under spinal anaesthesia done due to medical reasons-Malaria in Pregnancy. All the 3 children are alive and well.

Ct scan showed a chronic subdural hematoma (Figure 4) which was evacuated by burr holes. 


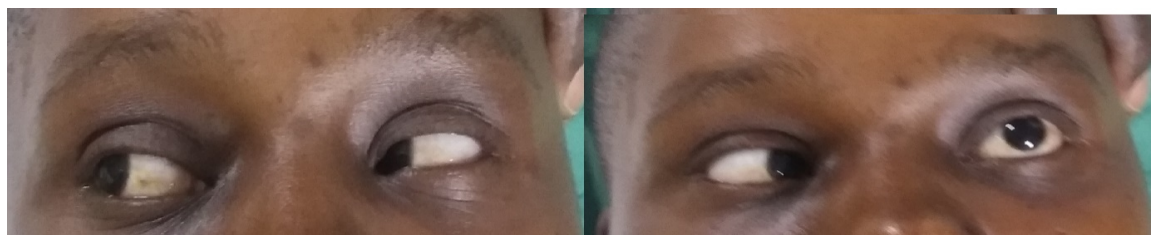

Figure 3. Left sided abducens palsy.

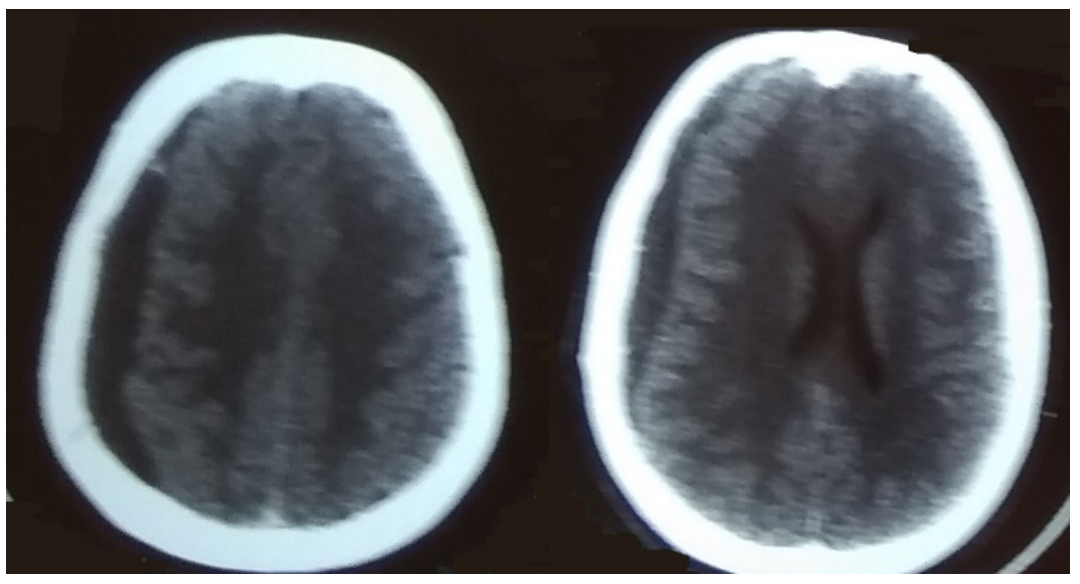

Figure 4. Chronic subdural hematoma with subfalcine herniation.

The post-operative course showed improvement of the double vision and patient was discharged home after $48 \mathrm{hrs}$.

Review in the clinic 4 weeks after evacuation revealed clinical resolution of the abducens nerve palsy (Figure 5).

\subsection{Case 3}

A 30-year-old female who presented with a 6-week history of headaches. This after an emergency caesarean section 6 weeks prior. The headache started $24 \mathrm{hrs}$ postop. It was a frontal headache not relieved by analgesics. Two weeks prior to admission had post prandial vomiting refractory to antiemetics. The symptoms progressed to include double vision and increased forgetfulness. On examination she was anxious and agitated, GCS 15/15, had no restriction of the extraocular motility but had bilateral papilloedema. CT scan imaging showed right sided chronic subdural heamatoma. She had burrholes done with resolution of the chronic subdural heamatoma (Figure 6).

\subsection{Case 4}

A 32-year-old female presented with intractable non postural headaches after caesarean section. Her antenatal profile was positive for HIV, with viral load well undetectable using ARVs for the past 4 years. The headaches were associated with nausea but no vomiting. Four weeks after caesarean section the obstetrician ordered a CT scan which showed a chronic subdural hematoma and referred to the neurosurgical service. On examination she had no focal deficits. Burrhole evacuation was done with resolution of symptoms (Figure 7). 


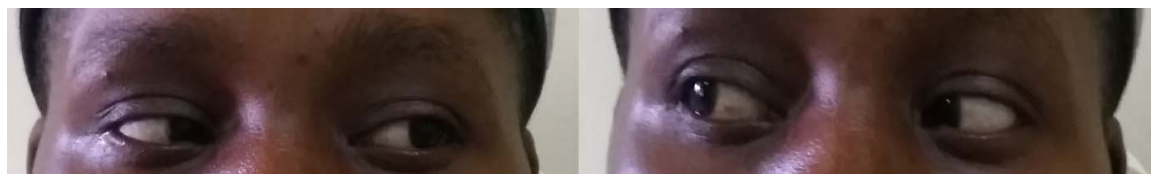

Figure 5. Resolution of left sided abducens nerve palsy one month after surgery.

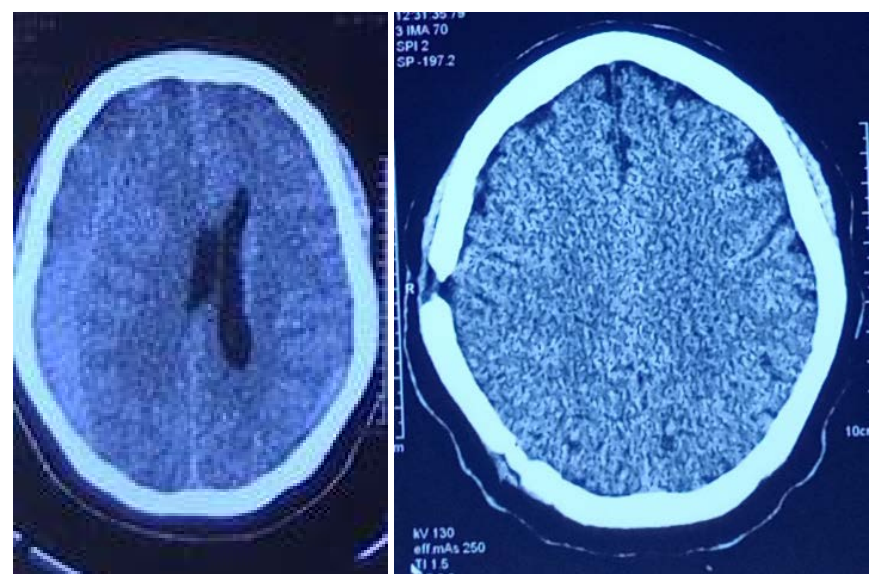

Figure 6. Right sided Chronic subdural hematoma with subfalcine herniation and resolution 6 weeks later.

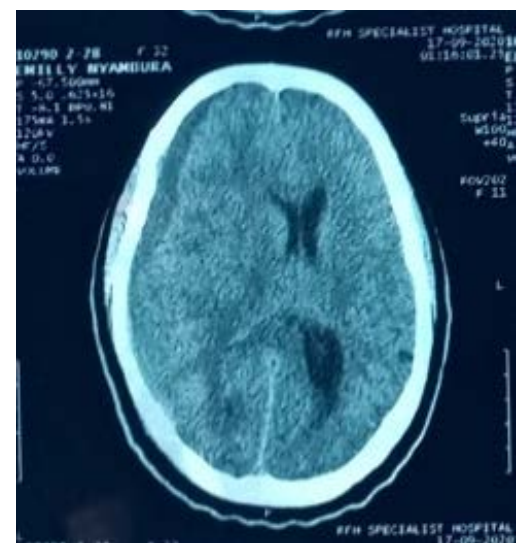

Figure 7. Right sided chronic subdural hematoma.

\section{Discussion}

Giving birth in Kenya is inherently dangerous with a high maternal mortality at 342 per 100,000 live births. Nine percent of births are delivered via caesarean section of 1.5 million births thus it's the most prevalent major surgical procedure done in Kenya. Caesarean births are often presented to expectant mothers as equal in alternative to vertex delivery with minimal complications [1].

Incidence of post-dural puncture headaches in Kenya following caesarean section has been estimated to be $20 \%$. The rate is much higher in quincke pointe compared to pencil point dural needles. This rate is higher than what has been quoted internationally at 7.75\% [2] [3].

In a review of 75 patients with post dural puncture, headaches occurred within $48 \mathrm{hrs}$ of surgery in $92 \%$ of the patients with the median duration being 5 days 
(range 2 - 10 days). Lybecker et al. graded the severity of the subdural headaches using a functional scale utilising the degree the patient was bed ridden during the day. Grade 1, which was graded Mild, patients had headaches but were not bed-ridden at any time of day. Grade 2 was moderate with the patient bed ridden part of the day. Grade 3 was bed ridden all the day with associated vestibular symptoms of nausea, vomiting, vertigo or dizziness [3].

Whether postdural puncture headaches present as a separate pathology or as an interlude to chronic subdural hematoma in these women is unknown. Chronic subdural hematoma as a complication of spinal anaesthesia is rare [4].

Chronic subdural hematomas arise from shearing of bridging veins that traverse the subarachnoid space. This is occasioned by a minor trauma that causes rapid acceleration and deceleration in a rostral caudal motion. Normally shearing after minor trauma does not occur as the brain parenchyma fits against the meningeal surface ensuring similar inertial properties when such a motion occurs. This property is provided for by the internal pressure dynamics of the skull contents, namely brain parenchyma, cerebrospinal fluid and the blood flowing through its vessels. Disease processes that contract the parenchymal surface from the fixed dural surfaces, therefore increasing risk of shearing of the vessels, do so by causing parenchymal loss or loss of CSF pressure [5].

Chronic subdural hematoma is therefore overwhelmingly more common in the elderly where brain atrophy is present. It is also a common complication of Cerebrospinal fluid diversion were over-shunting causes the brain to be lax and contract against the dural surface. In non-geriatric patients, up to $25 \%$ of patients developing CSDH were shown to have occult spinal CSF leaks in form of spinal cysts on MRI [6].

Since the only identifiable risk factor these patients presented with was spinal anaesthesia where the CSF dynamics were potentially altered, we propose that this is the cause of the chronic subdural hematoma in these two patients. With the large number of caesarean sections under spinal anaesthesia being done in Kenya, the contributions of chronic subdural hematoma to the maternal indices is unknown.

We therefore propose a bigger study where patients with persistent post-dural headaches will be followed up with imaging to determine the prevalence of chronic subdural hematoma in patients undergoing spinal anaesthesia.

\section{Informed Consent}

Informed consent in both cases was obtained in accordance to the World medical Association declaration of helsinki-ethical principles for medical research involving human subjects.

\section{Conflicts of Interest}

The authors declare no conflicts of interest regarding the publication of this paper. 


\section{References}

[1] National Bureau of Statistics (2015) Nairobi K. Republic of Kenya, Kenya Demographic and Health Survey 2014.

[2] Gisore, E., Mung'ayi, V. and Sharif, T. (2010) Incidence of Post Dural Puncture Headache Following Caesarean Section under Spinal Anaesthesia at the Aga Khan University Hospital, Nairobi. East African Medical Journal, 87, 227-230.

https://doi.org/10.4314/eamj.v87i6.63078

[3] Lybecker, H., Djernes, M. and Schmidt, J.F. (1995) Postdural Puncture Headache (PDPH): Onset, Duration, Severity, and Associated Symptoms: An Analysis of 75 Consecutive Patients with PDPH. Acta Anaesthesiologica Scandinavica, 39, 605-612. https://doi.org/10.1111/j.1399-6576.1995.tb04135.x

[4] Szeto, V., Kosirog, J. and Eilbert, W. (2018) Intracranial Subdural Hematoma after Epidural Anesthesia: A Case Report and Review of the Literature. International Journal of Emergency Medicine, 11, 36. https://doi.org/10.1186/s12245-018-0199-2

[5] Han, S.-B., Choi, S.-W., Song, S.-H., Youm, J.-Y., Koh, H.-S., Kim, S.-H., et al. (2014) Prediction of Chronic Subdural Hematoma in Minor Head Trauma Patients. Korean Journal of Neurotrauma, 10, 106. https://doi.org/10.13004/kjnt.2014.10.2.106

[6] Beck, J., Gralla, J., Fung, C., Ulrich, C.T., Schucht, P., Fichtner, J., et al. (2014) Spinal Cerebrospinal Fluid Leak as the Cause of Chronic Subdural Hematomas in Nongeriatric Patients. Journal of Neurosurgery, 121, 380-387.

https://doi.org/10.3171/2014.6.JNS14550 